\title{
Pancreatic Lipomatosis Picked Up on Imaging
}

\author{
GAURAV GUPTA, ${ }^{1}$ SANDEEP NIJ HAWAN, ${ }^{2}$ RAHUL VI RANI, ${ }^{3}$ SUBHASH NEPALIA ${ }^{4}$
}

A 55 year female with no co morbid illness presented with pain upper abdomen for three months. She complained of pain worsening after meals. Past medical history was unremarkable and she was not a smoker or alcohol consumer or diabetic. Her investigations revealed normal hematological parameters and liver function test. Serum amylase was $12 \mathrm{U} /$ L (20-90U/L) and serum lipase was 16U/L(40-200U/L) . Ultrasonography abdomen showed normal sized hyper echoic pancreas with rest of the study being normal. Computed tomography (CT) revealed fat replacement of pancreatic tissue leaving only a small amount of normal

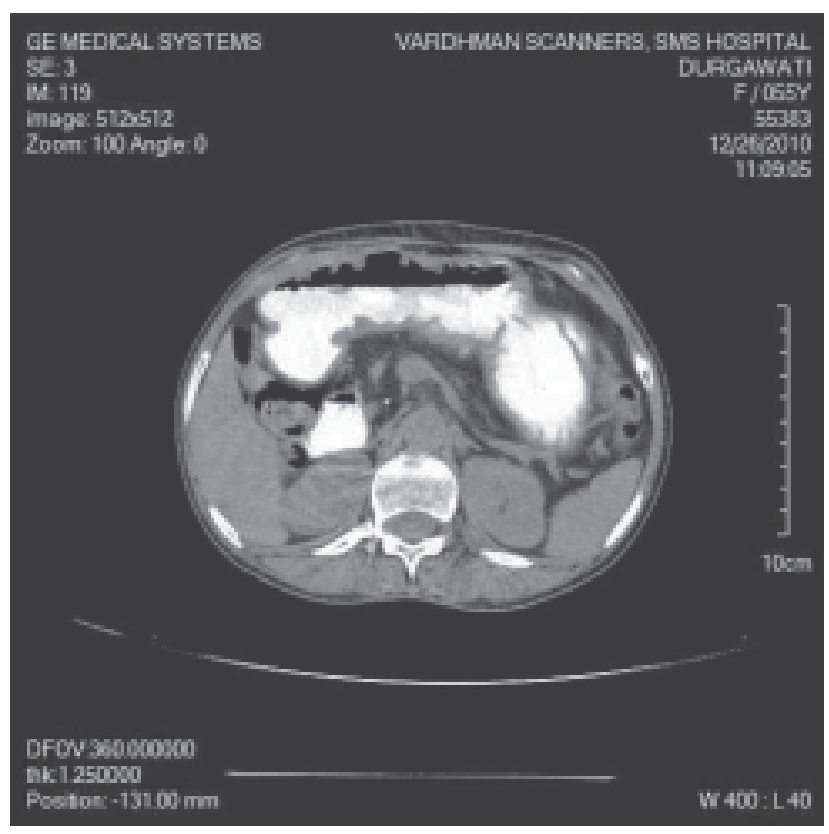

Fig.-1: Computed tomography image - fatty replacement of pancreas with sparing of small amount of parenchyma parenchyma around the main pancreatic duct (figure 1). Qualitative stool examination showed presence of fat globules. Based on these parameters a diagnosis of pancreatic lipomatosis with subclinical pancreatic exocrine insufficiency was made. She was managed with pancreatic enzyme therapy and became symptomatically better.

Lipomatosis of the pancreas is a rare lesion. Underlying pathogenesis is still unclear though it has been observed to be associated with obesity, ageing, diabetes mellitus, chronic pancreatitis, dietary deficiency, liver disease, viral illness, steroid therapy, congenital anomalies, occlusion of excretory ducts $^{1,2}$. Presentation varies from asymptomatic disease, pain abdomen, pancreatic insufficiency and features of predisposing conditions. Diagnosis is made by imaging. Ultrasonography shows hyper echoic pancreas but is less sensitive and have limitations. Computed tomography show normal or enlarged organ with fat replacement as hypodense area without normal parenchyma. MRI features are hyperintense signals on T1 and T2 images and null signal on short time inversion recovery weighted images ${ }^{3}$. Pancreatic function can be helpful in some cases. Liposarcoma of pancreas is a close differential diagnosis. Management of this condition is according to the predisposing factor and clinical presentation.

\section{Conflict of Interest: None}

\section{References:}

1. Mikihiko Yasuda, Yusuke Niina, Masahiko Uchida et al. A case of lipomatous pseudohypertrophy of the pancreas diagnosed by typical imaging. Journal of the Pancreas 2010; 11(4): 385-8

2. Boom EP, Van Der Sande FM, Tjon RT et al. Shwachman syndrome: CT and MRI diagnosis. J Comput Assit Tomogr $1993 ; 17: 474-6$

1. Department of gastroenterology, SMS Hospital Jaipur, Raj, India

2. Department of gastroenterology, SMS Hospital Jaipur, Raj, India

3. Department of Radiodiagnosis, SMS Hospital Jaipur, Raj, India

4. Department of gastroenterology, SMS Hospital Jaipur, Raj, India

Correspondence : Gaurav Gupta, Department of gastroenterology SMS Hospital, Jaipur (Raj) India Email- kumarggauravpgi@gmail.com 